\title{
A New Three-Dimensional Sliding Mode Guidance Law Variation with Finite Time
}

\section{Convergence}

\author{
Hyo-Sang Shin, Ke-Bo Li, and Antonios Tsourdos
}

\begin{abstract}
This paper develops a new three dimensional (3D) guidance law which guarantees the interception of manoeuvring targets in a finite time. The new guidance law accepts the concept that nullifying the line-of-sight (LOS) rate guarantees the interception of the target and its derivation is based on finite time sliding mode guidance. By using a 3D kinematic equation set constructed in a rotating coordinate system, the proposed guidance law alleviates an issue of general 3D guidance caused by the cross coupling effect between pitch and yaw planes. In theoretical analysis, finite time convergence of the new guidance law is proved and compared with that of a practical sliding mode guidance law. Characteristics such as energy consumption and convergence boundary layer are also theoretically analysed. Simulation results demonstrate that the new guidance law effectively intercepts manoeuvring targets in a finite time and analysis results are valid.
\end{abstract}

\section{Index Terms}

Sliding mode guidance, three dimensional pursuit, finite time convergence, manoeuvring target

\section{INTRODUCTION}

In the recent past, the sliding mode variable structure theory has drawn a great attention as a potential solution to the performance degradation issue in classical Proportional Navigation (PN) guidance laws against manoeuvring targets. As a result, there have been a large number of sliding mode guidance laws developed to effectively deal with missile guidance problems against agile targets with high manoeuvrability. However, most of these guidance laws provide only asymptotic or exponential stability. A guidance law with asymptotic or exponential stability typically drives the line-of-sight (LOS) rate to zero or to its small neighbourhood only as time approaches infinity.

Shtessel et al. [1], [2] proposed a smooth second-order sliding mode guidance law which can guarantee the finite time convergence. However, this guidance command can handle only sufficiently smooth uncertainty and

H.-S. Shin, and A. Tsourdos are with the Institute of Aerospace Sciences, SATM, Cranfield University, College Road, Cranfield, Bedfordshire, MK43 0AL, United Kingdom.

K.-B. Li is with Department of Aerospace, College of Aerospace Science and Technology, National University of Defense Technology, Changsha, Hunan, 410073, China 
disturbance, which might be impractical. Zhou et al. [3] recently proposed a two-dimensional (2D) finite time sliding mode guidance law (FTSMG) and its application in three-dimensional (3D) space. This guidance law was an extension of their previously proposed adaptive sliding mode guidance law (ASMG) [4] and can guarantee the finite time convergence and finite time stability in both the planar and 3D environments. Later, it was improved by considering the first-order autopilot lag of the missile system [5]. The guidance law in [5] is then extended to take impact angle constraints into account [6]. Wang and Wang [7] advanced a partial integrated missile guidance and control method with adaptive nonsingular terminal sliding mode control (ANTSM), such that finite time convergence can be guaranteed without the prior knowledge of the uncertainty and disturbance. Then, they extended this method to design guidance laws satisfying three-dimensional (3D) impact angle constraints [8].

The deduction and analysis about the 2D FTSMG in Ref. [3] are very sound and appropriate. Since the guidance command from this FTSMG is not a function of the LOS angular acceleration, the FTSMG proposed in Ref. [3] is found to be practical when compared with other finite time sliding mode guidance laws. Note that it is often difficult to measure or precisely estimate the LOS angular acceleration in practice. Rigorous and careful investigations on this guidance law led to improvement in construction of 3D FTSMG, i.e. development of a new guidance law.

The 3D FTSMG was also proposed in Ref. [3], which claimed that this was the first guidance law available with finite convergence in a 3D environment. Under the assumption that the cross coupling between the elevation and azimuth can be ignored, the 3D FTSMG was separately designed by constructing two 2D FTSMGs in the pitch and yaw planes of the missile in Ref. [3]. This is based on the 3D relative kinematic equation set established in the spherical coordinate system of the LOS with the origin fixed at the missile's centre of gravity [9]-[11]. Then, the authors proved the finite time stability of their 3D FTSMG considering the coupling effect. Although this approach has been commonly used, it might not be the most effective way to build 3D guidance laws for an interceptor: if possible, it would be desirable to design a 3D guidance law by directly considering 3D space without decoupling the space into two 2D planes. Moreover, this approach ignores the cross coupling effect between the two planes, which is valid only for small LOS angles and rates, in the design procedure. Extra complexity will be added to the guidance problem and the guidance performance could be degraded when LOS angles and their angular rates are not small enough.

In this paper, a 3D relative kinematic equation set established in a rotating LOS coordinate system [12] is considered to design 3D guidance directly from 3D space and mitigate the cross coupling issue. A few 3D guidance laws were already devised using this rotating LOS or similar coordinate system. Advantages of utilising this type of coordinate system were also demonstrated. For example, Li C. Y. et al. [13] and Li K. B. et al. [14], [15] used this kinematic equation set to study 3D differential geometric guidance command (DGGC). Tyan [16], [17] utilised another 3D relative kinematic equation set established in the modified polar coordinate system (MPC) of LOS to study the unified approach to guidance laws in 3D space and also the capture region of the generalised ideal proportional navigation (GIPN). Later, it was shown in Ref. [18] that the relative kinematic equation set used by Tyan [16], [17] is actually identical to the rotating LOS coordinate system which was used by Li C. Y. et al. [13] and Li K. B. et al. [14], [15]. 
This paper proposes a 3D FTSMG guidance law of which the commanded acceleration is directly constructed using the $3 \mathrm{D}$ relative kinematic equations in the rotating LOS coordinate system. The theoretical basis of the proposed guidance law is Lyapunov scalar differential inequality, like in Ref. [3]. Utilisation of Lyapunov scalar differential inequality will significantly reduce the complexity, compared with the case using the second order sliding mode guidance, as it is known that its complexity is comparable to that of the first-order sliding mode guidance law [3]. Furthermore, the proposed approach for the new guidance in this paper can resolve the issue with neglecting the cross coupling effect. Finite time convergence and performance of the new guidance law are theoretically analysed and compared with the original 3D FTSMG in Ref. [3]. Given the same engagement conditions and design parameters, the energy required in the proposed guidance law is smaller compared with the 3D FTSMG in Ref. [3]. Moreover, the boundary layer of the converging LOS rate in the new 3D FTSMG is smaller than in the original 3D FTSMG.

This paper consists of six sections. In Section II, the new guidance law is developed using the kinematic equation set constructed in the rotating coordinate system. Section III proves the finite time convergence of the proposed 3D FTSMG and its settling time is theoretically investigated. In Section IV, the characteristics of the new guidance law are analysed and compared with those of the 3D FTSMG in Ref. [3]. Section VI offers conclusions of this study after demonstrating analysis results through numerical simulations in Section V.

\section{PRELIMINARIES}

In this section, the original guidance law proposed in Ref. [3] will be first introduced. Then, we will introduce a new 3D FTSMG after discussing the rotating LOS coordinate system and a 3D relative kinematic equation set established in that coordinate system.

In Ref. [3], the spherical LOS coordinate system $(r, \theta, \phi)$ with the origin at the missile's gravity centre was used. The three unit vectors along the coordinate axes are denoted as $\left(\boldsymbol{e}_{r}, \boldsymbol{e}_{\theta}, \boldsymbol{e}_{\phi}\right)$. The relative dynamic equation set established in this coordinate system is given by:

$$
\left\{\begin{aligned}
\ddot{r}-r \dot{\phi}^{2}-r \dot{\theta}^{2} \cos ^{2} \phi & =a_{t r}-a_{m r}=a_{r} \\
(r \ddot{\theta}+2 \dot{r} \dot{\theta}) \cos \phi-2 r \dot{\phi} \dot{\theta} \sin \phi & =a_{t \theta}-a_{m \theta}=a_{\theta} \\
r \ddot{\phi}+2 \dot{r} \dot{\phi}+r \dot{\theta}^{2} \sin \phi \cos \phi & =a_{t \phi}-a_{m \phi}=a_{\phi}
\end{aligned}\right.
$$

where $r$ is the relative range, $\theta$ and $\phi$ the LOS azimuth and elevation angles, respectively, and $a$ the acceleration magnitude. Here, variables with subscripts " $m$ " and " $t$ " imply those variables of the missile and target, and variables with subscripts $r, \theta$, and $\phi$ mean projections of those variables on the three axes of the spherical LOS frame.

In Ref. [3], 3D FTSMG was realised by constructing two separate 2D FTSMGs in the pitch and yaw planes of the missile. The commanded accelerations in the two planes are obtained as:

$$
\left\{\begin{array}{l}
a_{m \theta}=-N \dot{r} \dot{\theta}+f_{\theta} \operatorname{sgn}(\dot{\theta})+\beta|\dot{\theta}|^{\eta} \operatorname{sgn}(\dot{\theta}) \\
a_{m \phi}=-N \dot{r} \dot{\phi}+f_{\phi} \operatorname{sgn}(\dot{\phi})+\beta|\dot{\phi}|^{\eta} \operatorname{sgn}(\dot{\phi})
\end{array}\right.
$$

where $N$ denotes the navigation constant whose value is bigger than $2, f_{\theta}$ and $f_{\phi}$ the bounds of $a_{t \theta}$ and $a_{t \phi}$, and $\beta>0$ and $\eta \in[0,1[$ constant guidance coefficients. 


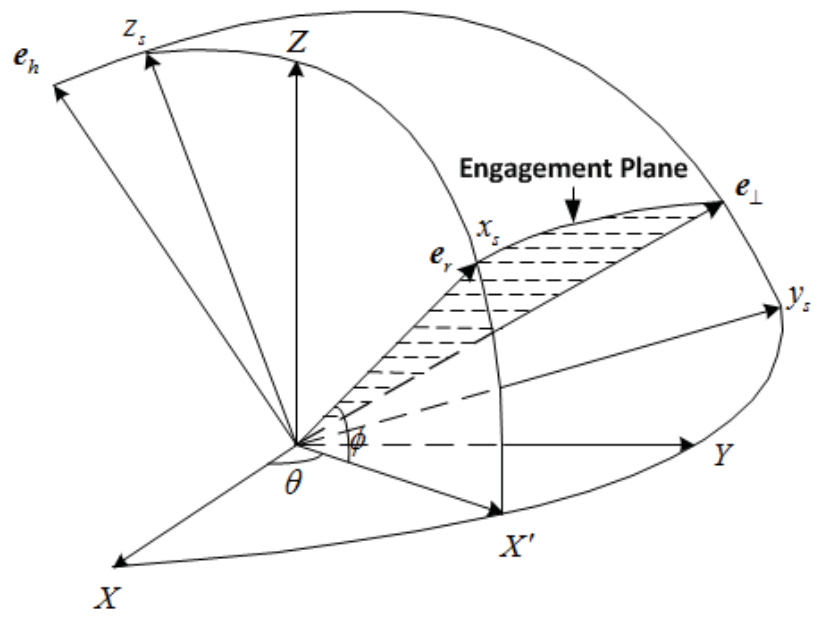

Fig. 1. Geometric relationship between spherical LOS coordinates and rotating LOS coordinates

From Eqn. (1), it is clear that, if $\theta$ and $\phi$ are small enough, the cross coupling in the pitch and yaw planes can be ignored, i.e., the relative motions in the two planes can be decoupled. However, if this small angle approximation is invalid, performance degradation of 3D FTSMG is inevitable due to the cross coupling effect.

Therefore, instead of spherical LOS coordinates, the LOS rotating coordinate system is utilised to develop a 3D FTSMG algorithm in this paper. This approach mitigates the cross coupling issue in Ref. [3], and it significantly simplifies the construction of the guidance law. The three unit vectors along the coordinate axes of the rotating LOS frame are denoted as $\left(\boldsymbol{e}_{r}, \boldsymbol{e}_{\perp}, \boldsymbol{e}_{h}\right): \boldsymbol{e}_{r}$ is the unit vector along the relative range vector, $\boldsymbol{e}_{h}$ the unit vector along $\dot{\boldsymbol{e}}_{r}$, and $\boldsymbol{e}_{\perp}$ the normal direction of the engagement plane such that. Note that the engagement plane is spanned by $\boldsymbol{e}_{r}$ and $\boldsymbol{e}_{\perp}$ as shown in Fig. 1 and $\boldsymbol{e}_{h}$ follows the right-hand rule such that:

$$
\boldsymbol{e}_{h}=\boldsymbol{e}_{r} \times \boldsymbol{e}_{\perp}
$$

Let $\boldsymbol{\omega}_{L O S c o o r}$ and $\boldsymbol{\omega}_{s}$ denote the angular velocity of the rotating axes $\left(\boldsymbol{e}_{r}, \boldsymbol{e}_{\perp}, \boldsymbol{e}_{h}\right)$ and the rotational rate of $\boldsymbol{r}$. Then, $\boldsymbol{\omega}_{L O S c o o r}$ can be represented as:

$$
\boldsymbol{\omega}_{\text {LOScoor }}=\boldsymbol{\omega}_{\boldsymbol{s}}+\boldsymbol{\Omega}_{s}=\omega_{s} \boldsymbol{e}_{h}+\Omega_{s} \boldsymbol{e}_{r}
$$

where $\omega_{s} \geq 0$ is the absolute angular speed of LOS, $\boldsymbol{\Omega}_{s}$ is the component of $\omega_{L O S c o o r}$ along $\boldsymbol{r}$, and $\Omega_{s}$ denotes the angular speed of the engagement plane. Therefore, the following property holds [12], [15], [18]:

$$
\left\{\begin{array}{l}
\dot{\boldsymbol{e}}_{r}=\omega_{s} \boldsymbol{e}_{\perp} \\
\dot{\boldsymbol{e}}_{\perp}=-\omega_{s} \boldsymbol{e}_{r}+\Omega_{s} \boldsymbol{e}_{h} \\
\dot{\boldsymbol{e}}_{h}=-\Omega_{s} \boldsymbol{e}_{\perp}
\end{array}\right.
$$


The relative kinematic equation set in rotating LOS frame is then obtained as:

$$
\left\{\begin{array}{r}
\ddot{r}-r \omega_{s}^{2}=a_{t r}-a_{m r} \\
r \dot{\omega}_{s}+2 \dot{r} \omega_{s}=a_{t \perp}-a_{m \perp} \\
r \omega_{s} \Omega_{s}=a_{t h}-a_{m h}
\end{array}\right.
$$

where subscripts $r, \perp, h$ on variables represent projections of those variables onto the three axes of $\left(\boldsymbol{e}_{r}, \boldsymbol{e}_{\perp}, \boldsymbol{e}_{h}\right)$. The first two equations in Eqn. (6) describe the relative motion in the engagement plane and the third equation represents the rotational principle of the engagement plane. As shown in Eqn. (6), the first two equations can be decoupled from the third one. For more details, the reader is referred to [12], [15], [18].

Fig. 1 illustrates the geometric relationship between $\left(\boldsymbol{e}_{r}, \boldsymbol{e}_{\theta}, \boldsymbol{e}_{\phi}\right)$ and $\left(\boldsymbol{e}_{r}, \boldsymbol{e}_{\perp}, \boldsymbol{e}_{h}\right)$. As shown in Fig. 1, the spherical LOS coordinates, $\left(\boldsymbol{e}_{r}, \boldsymbol{e}_{\theta}, \boldsymbol{e}_{\phi}\right)$, can be obtained by firstly rotating the inertial coordinates $(\boldsymbol{X}, \boldsymbol{Y}, \boldsymbol{Z})$ around $\boldsymbol{Z}$ with angle $\theta$ and secondly rotating the rotated coordinates around $\boldsymbol{y}_{s}$ with angle $-\phi$ such that:

$$
\left[\begin{array}{l}
\boldsymbol{e}_{r} \\
\boldsymbol{e}_{\theta} \\
\boldsymbol{e}_{\phi}
\end{array}\right]:=\left[\begin{array}{l}
\boldsymbol{x}_{s} \\
\boldsymbol{y}_{s} \\
\boldsymbol{z}_{s}
\end{array}\right]=\left[\begin{array}{ccc}
\cos \theta \cos \phi & \sin \theta \cos \phi & \sin \phi \\
-\sin \theta & \cos \theta & 0 \\
-\cos \theta \sin \phi & -\sin \theta \sin \phi & \cos \phi
\end{array}\right]\left[\begin{array}{l}
\boldsymbol{X} \\
\boldsymbol{Y} \\
\boldsymbol{Z}
\end{array}\right]
$$

The angular velocity of spherical LOS coordinates, $\boldsymbol{\omega}_{L O S c o o r, s}$, can be represented as:

$$
\boldsymbol{\omega}_{L O S \operatorname{coor}, s}=\dot{\theta} \boldsymbol{Z}-\dot{\phi} \boldsymbol{y}_{s}=\dot{\theta} \sin \phi \boldsymbol{x}_{s}-\dot{\phi} \boldsymbol{y}_{s}+\dot{\theta} \cos \phi \boldsymbol{z}_{s}
$$

The angular velocity of $\boldsymbol{e}_{r}$ is given by:

$$
\begin{aligned}
\dot{\boldsymbol{e}}_{r} & :=\dot{\boldsymbol{x}}_{s}=\boldsymbol{\omega}_{\text {LOScoor }, s} \times \boldsymbol{x}_{s}=\left(\dot{\theta} \sin \phi \boldsymbol{x}_{s}-\dot{\phi} \boldsymbol{y}_{s}+\dot{\theta} \cos \phi \boldsymbol{z}_{s}\right) \times \boldsymbol{x}_{s} \\
& =\dot{\theta} \cos \phi \boldsymbol{y}_{s}+\dot{\phi} \boldsymbol{z}_{s}
\end{aligned}
$$

From the definitions of $\omega_{s}$ and $\left(\boldsymbol{e}_{r}, \boldsymbol{e}_{\perp}, \boldsymbol{e}_{h}\right)$, we have:

$$
\left\{\begin{array}{l}
\omega_{s}=\sqrt{(\dot{\theta} \cos \phi)^{2}+\dot{\phi}^{2}} \\
\boldsymbol{e}_{\perp}=\left(\dot{\theta} \cos \phi \boldsymbol{y}_{s}+\dot{\phi} \boldsymbol{z}_{s}\right) / \omega_{s}=\left(\dot{\theta} \cos \phi \boldsymbol{e}_{\theta}+\dot{\phi} \boldsymbol{e}_{\phi}\right) / \omega_{s} \\
\boldsymbol{e}_{h}=\left(-\dot{\phi} \boldsymbol{x}_{s}+\dot{\theta} \cos \phi \boldsymbol{z}_{s}\right) / \omega_{s}=\left(-\dot{\phi} \boldsymbol{e}_{r}+\dot{\theta} \cos \phi \boldsymbol{e}_{\phi}\right) / \omega_{s}
\end{array}\right.
$$

The second equation in Eqn. (6) can be rewritten as:

$$
\dot{\omega}_{s}=-\frac{2 \dot{r}}{r} \omega_{s}-\frac{a_{m \perp}}{r}+\frac{a_{t \perp}}{r}
$$

Accepting the typical guidance concept that nullifying the LOS angular speed $\omega_{s}$ results in the interception of the target, the guidance problem is to find the commanded acceleration, $\boldsymbol{a}_{m c}$, which drives $\omega_{s}$ in Eqn. (11) to zero or its small neighbourhood in a finite time. Similar to the 3D FTSMG in Ref. [3], to guarantee the finite convergence of the LOS angular speed, the commanded acceleration of new 3D FTSMG is proposed as:

$$
\begin{aligned}
\boldsymbol{a}_{m c} & =a_{m \perp} \boldsymbol{e}_{\perp}, \\
a_{m \perp} & =-N \dot{r} \omega_{s}+f_{\perp} \operatorname{sgn}\left(\omega_{s}\right)+\beta \omega_{s}^{\eta} \operatorname{sgn}\left(\omega_{s}\right)
\end{aligned}
$$


where $N>2, \beta>0$, and $\eta \in\left[0,1\left[\right.\right.$ are again constant, and $f_{\perp}$ is the bound of the target acceleration component, $a_{t \perp}$, thus:

$$
\left|a_{t \perp}\right| \leq f_{\perp}
$$

Since $\omega_{s}$ is non-negative from its definition, a signum function does not need to be introduced in Eqn. (12):

$$
\begin{aligned}
\boldsymbol{a}_{m c} & =a_{m \perp} \boldsymbol{e}_{\perp}, \\
a_{m \perp} & =-N \dot{r} \omega_{s}+f_{\perp}+\beta \omega_{s}^{\eta}
\end{aligned}
$$

Note that the unit vector $\boldsymbol{e}_{\perp}$ is given in Eqn. (5). The derivation of the proposed guidance law and its finite time convergence will be investigated in the following section.

\section{3D Finite Time Convergence Guidance}

The commanded guidance of the proposed 3D FTSMG guidance law is obtained to guarantee the finite time convergence. Therefore, this session focuses to derive the 3D FTSMG guidance law and prove its finite time convergence.

Let us first introduce a lemma, which will be essential to the following discussions.

Lemma 1. Consider the nonlinear system described as:

$$
\dot{\boldsymbol{x}}=g(\boldsymbol{x}, t), \quad g(\mathbf{0}, t)=0, \quad \boldsymbol{x} \in R^{n}
$$

where $g: U_{0} \times R \rightarrow R^{n}$ is continuous on $U_{0} \times R$ and $U_{0}$ is an open neighbourhood of the origin $\boldsymbol{x}=\mathbf{0}$. Suppose that there is a $C^{1}$ (continuously differentiable) function $V(x, t)$ defined in a neighbourhood $\hat{U} \subset R^{n}$ of the origin, and that there are real numbers $\alpha>0$ and $0<\lambda<1$, such that $V(x, t)$ is positive definite on $\hat{U}$ and that $\dot{V}(x, t)+\alpha V^{\lambda}(x, t) \leq 0$ on $\hat{U}$. Then, the zero solution of the system in Eqn. (15) is finite time stable. The settling time is given by:

$$
t_{s} \leq \frac{V^{1-\lambda}\left(\boldsymbol{x}_{0}, 0\right)}{\alpha(1-\lambda)}
$$

where $\boldsymbol{x}_{0}=\boldsymbol{x}(0)$.

Proof. Note that this lemma is identical to Lemma 1 in Ref. [3]. The proof of Lemma 1 is briefly given here, but it is indifferent from that in Ref. [3].

From the assumption $\dot{V}(\boldsymbol{x}, t)+\alpha V^{\lambda}(\boldsymbol{x}, t) \leq 0$ on $\hat{U}$, we have:

$$
\frac{d V(x, t)}{V^{\lambda}(x, t)} \leq-\alpha d t, \quad \forall t \geq 0
$$

As $V(x, t)$ is positive definite on $\hat{U}$, it is clear that:

$$
\left\{\begin{array}{l}
V^{1-\lambda}(\boldsymbol{x}, t) \leq-\alpha(1-\lambda) t+V^{1-\lambda}\left(\boldsymbol{x}_{0}, 0\right), \text { for } 0 \leq t<t_{s} \\
V(x, t)=0, \quad \text { for } t \geq t_{s}
\end{array}\right.
$$


where $t_{s}$ denotes the settling time, which can be obtained as:

$$
t_{s} \leq \frac{V^{1-\lambda}\left(\boldsymbol{x}_{0}, 0\right)}{\alpha(1-\lambda)}
$$

This completes the proof.

As stated, we accept the typical guidance concept, i.e. the interception of the target can be achieved by nullifying the LOS angular speed $\omega_{s}$. From this concept, a sliding surface $s$, can be defined as:

$$
s=\omega_{s}=0
$$

We can then define a continuous Lyapunov function, $V$, as:

$$
V=s^{2}=\omega_{s}^{2}
$$

Taking the first time derivative of $V$ gives:

$$
\dot{V}=2 \omega_{s} \dot{\omega}_{s}
$$

Substituting Eqn. (11) into Eqn. (22) yields:

$$
\dot{V}=2 \frac{\omega_{s}}{r}\left(-2 \dot{r} \omega_{s}-a_{m \perp}+a_{t \perp}\right)
$$

We designed the commanded acceleration such that $\dot{V}$ in Eqn. (23) holds the conditions in Lemma 1, to guarantee the finite convergence of the new 3D FTSMG.

Now, based on the proposed commanded acceleration given in Eqn. (14), let us prove the finite convergence of the proposed 3D FTSMG.

Theorem 1. Consider the nonlinear system of Eqn. (1). Suppose the following assumptions on the engagement condition are satisfied:

$$
\dot{r}(t)<0, \quad \forall t>0
$$

Then, the proposed guidance law summarised in Eqn. (14) nullifies the LOS angular rate in a finite time.

Proof. A continuous Lyapunov function is defined in Eqn. (21). Given the commanded acceleration of the proposed guidance described in Eqn. (14), the first time derivative of the Lyapunov-like function is obtained as:

$$
\dot{V}=\frac{2}{r}\left[\omega_{s}\left(a_{t \perp}-f_{\perp}\right)-\beta \omega_{s}^{\eta+1}+(N-2) \dot{r} \omega_{s}^{2}\right]
$$

For $N>2$, from Eqns. (13) and (24), it is then clear that:

$$
\dot{V} \leq-\frac{2 \beta}{r} \omega_{s}^{\eta+1} \leq 0
$$

Note that the condition defined in Eqn. (24) is generally met during the engagement. According to this engagement condition, $r_{0} \geq r(t)$ for all $t$. Here, the subscript " 0 " on a parameter means its initial condition. Hence, it is trivial that:

$$
\dot{V} \leq-\frac{2 \beta}{r_{0}} \omega_{s}^{\eta+1}
$$


Hence:

$$
\dot{V} \leq-\frac{2 \beta}{r_{0}} V^{\frac{\eta+1}{2}}
$$

In the proposed guidance law, we have $\beta>0$, and $\eta \in\left[0,1\left[\right.\right.$. Therefore, it is clear that $2 \beta / r_{0}=$ const. $>0$ and $0.5 \leq(\eta+1) / 2<1$. Therefore, from Lemma 1 , the finite time convergence under the proposed guidance law is proven.

The settling time of the LOS angular speed of the proposed guidance law can be also obtained from Lemma 1 .

Proposition 1. The settling time, $t_{s}$, of the Lyapunov function represent the convergence speed. The settling time, $t_{s}$, of the proposed guidance law holds the following inequality:

$$
t_{s} \leq \frac{r_{0} \omega_{s 0}^{1-\eta}}{\beta(1-\eta)}
$$

and this inequality condition is identical to that of the 3D FTSMG in Ref. [3].

Proof. From Eqn. (28) and Lemma 1, it is clear that Eqn. (29). The settling time of the 3D FTSMG in Ref. [3] satisfies:

$$
t_{s} \leq \frac{r_{0}\left(\dot{\theta}_{0}^{2} \cos ^{2} \phi_{0}+\dot{\phi}_{0}^{2}\right)^{\frac{1-\eta}{2}}}{\beta(1-\eta)}
$$

This inequality condition is identical to the settling time inequality condition of the proposed approach in Eqn. (29) since $\omega_{s 0}=\sqrt{\dot{\theta}_{0}^{2} \cos ^{2} \phi_{0}+\dot{\phi}_{0}^{2}}$.

Now, let's investigate the properties of the convergence speed of the proposed 3D FTSMG algorithm.

Remark 1. The convergence speed of the LOS angular rate can is most likely to be increased as the value of $\beta$ increases. Furthermore, the convergence speed is likely to be increased as the value of $\eta$ decreases when $\omega_{s 0} \leq$ $e(\approx 2.7183) \mathrm{rad} / \mathrm{s}$.

Proof. From the settling time in Eqn. (29), it is clear that the convergence rate of $\omega_{s}$ is most likely to be increased as the value of $\beta$ increases. From Eqn. (29), the derivative of the settling time bound w.r.t. $\eta$ is obtained as:

$$
\frac{d}{d \eta}\left(\frac{r_{0} \omega_{s 0}^{1-\eta}}{\beta(1-\eta)}\right)=\left(1+\eta \ln \omega_{s 0}-\ln \omega_{s 0}\right) \frac{r_{0} \omega_{s 0}^{1-\eta}}{\beta(1-\eta)}
$$

Hence, we have:

$$
\frac{d}{d \eta}\left(\frac{r_{0} \omega_{s 0}^{1-\eta}}{\beta(1-\eta)}\right) \geq 0, \quad \text { for } \omega_{s 0} \leq e^{\frac{1}{1-\eta}}
$$

The minimum value of $\omega_{s 0}$ holding the non-negative derivative of the bound is $e$. Therefore, for $\omega_{s 0} \leq e$, the derivative of the settling time is non-negative. This indicates the convergence speed is likely to be increased as $\eta$ decreases.

It is often difficult to predict the direction and pattern of the target manoeuvres. However, it might be still possible to estimate the maximum target acceleration $a_{t \max }$. In this case, $f_{\perp}$ in Eqn. (14) could be set to be bigger than or equal to the maximum target acceleration $a_{t \max }$. 
If the acceleration components of the target is precisely measured or estimated, $f_{\perp}$ can be replaced by $a_{t \perp}$ in Eqn. (14). Then, the commanded acceleration can be represented as:

$$
\begin{aligned}
& \boldsymbol{a}_{m c}=a_{m \perp} \boldsymbol{e}_{\perp}, \\
& a_{m \perp}=-N \dot{r} \omega_{s}+a_{t \perp}+\beta \omega_{s}^{\eta}
\end{aligned}
$$

This still guarantees the finite time convergence.

Proposition 2. Suppose that the target acceleration can be precisely measured or estimated and the engagement condition given in Eqn. (24) holds. Then, the 3D FTSMG with the commanded acceleration defined in Eqn. (33) holds the finite time convergence of the LOS angular rate and its settling time is bounded as in Eqn. (29).

Proof. Substituting $a_{m \perp}$ in Eqn. (33) into Eqn. (23) gives:

$$
\dot{V}=\frac{2}{r}\left[(N-2) \dot{r} \omega_{s}^{2}-\beta \omega_{s}^{\eta+1}\right]
$$

From the engagement condition defined in Eqn. (24), it is clear that:

$$
\dot{V} \leq-\frac{2 \beta}{r_{0}} V^{\frac{\eta+1}{2}}
$$

This completes the proof.

\section{Characteristics OF THe New 3D FTSMG}

In this section, the characteristics of the proposed 3D FTSMG will be compared with that of the original FTSMG in [3].

Unlike the approach proposed in this paper, Ref. [3] decoupled the two planes and obtained the commanded acceleration, ignoring the coupling between the two plane. This commanded acceleration vector of the 3D approach in Ref. [3], which is denoted as $\boldsymbol{a}_{\text {old }}$ is given by:

$$
\boldsymbol{a}_{\text {old }}=\left[-N \dot{r} \dot{\theta}+f_{\theta} \operatorname{sgn}(\dot{\theta})+\beta|\dot{\theta}|^{\eta} \operatorname{sgn}(\dot{\theta})\right] \boldsymbol{e}_{\theta}+\left[-N \dot{r} \dot{\phi}+f_{\phi} \operatorname{sgn}(\dot{\phi})+\beta|\dot{\phi}|^{\eta} \operatorname{sgn} \dot{\phi}\right] \boldsymbol{e}_{\phi},
$$

Then, its finite time stability is proven by reconsidering the coupling effect between the two planes. This could unnecessarily increase complexity in obtaining the commanded acceleration vector and the analysis of the stability. Previous Section confirms that complexity in the derivation and analysis of the 3D FTSMG is significantly reduced in our approach. From this difference between the original 3D FTSMG in Ref. [3] and newly proposed one, it is clear that we should investigate the effect of neglecting the coupling effect between the kinematics in the two plane.

From Eqns. (2) and (14), the original commanded acceleration in [3] is in the engagement plane if

$$
a_{m \theta} \dot{\phi}=a_{m \phi} \dot{\theta} \cos \phi
$$

However, this condition is seldom satisfied. If the commanded acceleration is not in the engagement plane, there exists a projection on $\boldsymbol{e}_{h}$, i.e., $a_{m h}$. From Eqn. (6), it is clear that $a_{m h}$ results in rotational motion of engagement plane which has no direct influence on the change in $\omega_{s}$. This implies that the 3D approach developed in Ref. [3] 
may cause missile manoeuvres unnecessary for the target interception and consequently waste energy. On the other hand, the commanded acceleration, $\boldsymbol{a}_{m c}$, in Eqn. (14) is always aligned on the engagement plane. Therefore, given the same engagement conditions and design parameters, the proposed guidance law in this paper could require smaller energy for the target interception in 3D space compared with 3D FTSMG in Ref. [3], especially when the small angle approximation on $\phi$ is invalid. This can be validated from following Theorem.

Theorem 2. Let us denote the commanded acceleration vectors of the 3D approach in Ref. [3] and the proposed $3 D$ FTSMG in this paper as $\boldsymbol{a}_{\text {old }}$ and $\boldsymbol{a}_{\text {new }}$, respectively. Then, $\boldsymbol{a}_{\text {old }}$ is given in Eqn. (35) and $\boldsymbol{a}_{\text {new }}$ is obtained as:

$$
\boldsymbol{a}_{n e w}=\left(-N \dot{r} \omega_{s}+f_{\perp}+\beta \omega_{s}^{\eta}\right) \boldsymbol{e}_{\perp}
$$

For fair comparison, suppose that $f_{\theta}, f_{\phi}$, and $f_{\perp}$ are all set to be equal to $a_{\text {tmax }}$. Then, given the same engagement conditions, the magnitude of the commanded acceleration of the proposed approach is smaller than that of the original in Ref. [3], if the design parameters, $N, \beta$, and $\eta$, are set to be identical in both the guidance approaches.

Proof. The magnitude squared of the commanded acceleration of the original approach is given by:

$$
\begin{aligned}
\left\|\boldsymbol{a}_{\text {old }}\right\|^{2}= & N^{2} \dot{r}^{2}\left(\dot{\theta}^{2}+\dot{\phi}^{2}\right)-2 N \dot{r}\left(f_{\theta}|\dot{\theta}|+f_{\phi}|\dot{\phi}|\right)-2 N \dot{r} \beta\left(|\dot{\theta}|^{\eta+1}+|\dot{\phi}|^{\eta+1}\right) \\
& +\left(f_{\theta}+\beta|\dot{\theta}|^{\eta}\right)^{2}+\left(f_{\phi}+\beta|\dot{\phi}|^{\eta}\right)^{2}
\end{aligned}
$$

and that of the new approach is obtained as:

$$
\left\|\boldsymbol{a}_{\text {new }}\right\|^{2}=N^{2} \dot{r}^{2} \omega_{s}^{2}-2 N \dot{r} \omega_{s} f_{\perp}-2 N \dot{r} \beta \dot{\omega}_{s}^{\eta+1}+\left(f_{\perp}+\beta\left|\dot{\omega}_{s}\right|^{\eta}\right)^{2} .
$$

The proof will be completed by showing that:

$$
\left\|\boldsymbol{a}_{\text {new }}\right\|^{2} \leq\left\|\boldsymbol{a}_{\text {old }}\right\|^{2}
$$

The first term on the right side of Eqn. (38) satisfies the following inequality:

$$
N^{2} \dot{r}^{2}\left(\dot{\theta}^{2}+\dot{\phi}^{2}\right) \geq N^{2} \dot{r}^{2}\left(\dot{\theta}^{2} \cos ^{2} \phi+\dot{\phi}^{2}\right)=N^{2} \dot{r}^{2} \omega_{s}^{2}
$$

Note that $f_{\perp}$ is the bound of the effective target evasion manoeuvre, i.e. the bound of the target acceleration on the direction of $\boldsymbol{e}_{\perp}$. Since $f_{\theta}, f_{\phi}$, and $f_{\perp}$ are set to be identical to $a_{\text {tmax }}$, from the definition of $\omega_{s}$, it is clear that:

$$
\omega_{s} f_{\perp} \leq f_{\theta}|\dot{\theta}|+f_{\phi}|\dot{\phi}|
$$

Setting

$$
\cos \zeta:=\frac{\dot{\theta} \cos \phi}{\omega_{s}}, \quad \sin \zeta:=\frac{\dot{\phi}}{\omega_{s}}
$$


it is clear that

$$
\begin{aligned}
\frac{|\dot{\theta}|^{\eta+1}+|\dot{\phi}|^{\eta+1}}{\omega_{s}^{\eta+1}} & \geq \frac{|\dot{\theta}|^{\eta}\left|\frac{\dot{\theta} \cos \phi}{\omega_{s}}\right|+|\dot{\phi}|^{\eta}\left|\frac{\dot{\phi}}{\omega_{s}}\right|}{\omega_{s}^{\eta}} \\
& \geq\left|\frac{\dot{\theta} \cos \phi}{\omega_{s}}\right|^{\eta+1}+\left|\frac{\dot{\phi}}{\omega_{s}}\right|^{\eta+1} \\
& =|\cos \zeta|^{\eta+1}+|\sin \zeta|^{\eta+1} \\
& \geq \cos ^{2} \zeta+\sin ^{2} \zeta=1
\end{aligned}
$$

where $\omega_{s} \geq 0$ by the definition. From Eqn. (44), we can take the following two inequalities :

$$
\begin{aligned}
& 1 \leq \frac{|\dot{\theta}|^{\eta}\left|\frac{\dot{\theta} \cos \phi}{\omega_{s}}\right|+|\dot{\phi}|^{\eta}\left|\frac{\dot{\phi}}{\omega_{s}}\right|}{\omega_{s}^{\eta}} \\
& 1 \leq \frac{|\dot{\theta}|^{\eta+1}+|\dot{\phi}|^{\eta+1}}{\omega_{s}^{\eta+1}}
\end{aligned}
$$

Hence, it is clear that:

$$
\begin{aligned}
\omega_{s}^{\eta} & \leq|\dot{\theta}|^{\eta}\left|\frac{\dot{\theta} \cos \phi}{\omega_{s}}\right|+|\dot{\phi}|^{\eta}\left|\frac{\dot{\phi}}{\omega_{s}}\right| \\
\omega_{s}^{\eta+1} & \leq|\dot{\theta}|^{\eta+1}+|\dot{\phi}|^{\eta+1}
\end{aligned}
$$

From Eqn. (46) and

$$
\left(\frac{\dot{\theta} \cos \phi}{\omega_{s}}\right)^{2}+\left(\frac{\dot{\phi}}{\omega_{s}}\right)^{2}=1,
$$

the last two terms on the right side of Eqn. (38) meet the following inequality condition:

$$
\begin{aligned}
\left(f_{\theta}+\beta|\dot{\theta}|^{\eta}\right)^{2}+\left(f_{\phi}+\beta|\dot{\phi}|^{\eta}\right)^{2} & =\left[\left(f_{\theta}+\beta|\dot{\theta}|^{\eta}\right)^{2}+\left(f_{\phi}+\beta|\dot{\phi}|^{\eta}\right)^{2}\right]\left[\left(\frac{\dot{\theta} \cos \phi}{\omega_{s}}\right)^{2}+\left(\frac{\dot{\phi}}{\omega_{s}}\right)^{2}\right] \\
& \geq\left[\left(f_{\theta}+\beta|\dot{\theta}|^{\eta}\right)\left|\frac{\dot{\theta} \cos \phi}{\omega_{s}}\right|+\left(f_{\phi}+\beta|\dot{\phi}|^{\eta}\right)\left|\frac{\dot{\phi}}{\omega_{s}}\right|\right]^{2} \\
& \geq\left(f_{\theta}\left|\frac{\dot{\theta} \cos \phi}{\omega_{s}}\right|+f_{\phi}\left|\frac{\dot{\phi}}{\omega_{s}}\right|+\beta|\dot{\theta}|^{\eta}\left|\frac{\dot{\theta} \cos \phi}{\omega_{s}}\right|+\beta|\dot{\phi}|^{\eta}\left|\frac{\dot{\phi}}{\omega_{s}}\right|\right)^{2} \\
& \geq\left(f_{\perp}+\beta \omega_{s}^{\eta}\right)^{2} .
\end{aligned}
$$

Since $\dot{r}<0$ during the engagement, from Eqns. (41), (42), (47), and (49), it is trivial that Eqn. (40) holds.

In practice, signum functions in the sliding mode control can cause undesirable chattering in the commanded acceleration. Although there is no signum function introduced in the commanded acceleration of the proposed $3 \mathrm{D}$ FTSMG, the chattering problem can happen when the LOS rate is small and close to zero. Small LOS rates may result in frequent changes in the direction of $\boldsymbol{e}_{\perp}$ as shown in the second equation of Eqn. (10) and consequently cause chattering in the commanded acceleration. It is well known that an effective approach to mitigate the chattering 
problem is to replace the signum function with a saturation or sigmoid function [6]. Introducing a saturation function to the proposed 3D FTSMG yields:

$$
\boldsymbol{a}_{m c}=a_{m \perp} \boldsymbol{e}_{\perp}=\left[-N \dot{r} \omega_{s}+\left(f_{\perp}+\beta \omega_{s}^{\eta}\right) \operatorname{sat}\left(\omega_{s}\right)\right] \boldsymbol{e}_{\perp},
$$

where, for a small $\delta>0, \operatorname{sat}\left(\omega_{s}\right)$ is given by:

$$
\operatorname{sat}\left(\omega_{s}\right)= \begin{cases}1, & \omega_{s}>\delta \\ \omega_{s} / \delta, & \omega_{s} \leq \delta .\end{cases}
$$

Remark 2. When $\omega_{s}>\delta$, Eqn. (50) is identical to Eqn. (14). Therefore, the proposed guidance command in Eqn. (50) drives the LOS angular speed to a boundary layer $\left|\omega_{s}\right| \leq \delta$.

Proposition 3. In the original $3 D F T S M G$, the commanded accelerations with the saturation function are given by:

$$
\left\{\begin{array}{c}
a_{m \theta}=-N \dot{r} \dot{\theta}+f_{\theta} \operatorname{sat}(\dot{\theta})+\beta|\dot{\theta}|^{\eta} \operatorname{sat}(\dot{\theta}) \\
a_{m \phi}=-N \dot{r} \dot{\phi}+f_{\phi} \operatorname{sat}(\dot{\phi})+\beta|\dot{\phi}|^{\eta} \operatorname{sat}(\dot{\phi})
\end{array}\right.
$$

where:

$$
\operatorname{sat}(\dot{\theta})=\left\{\begin{array}{ll}
1, & \dot{\theta}>\delta \\
\dot{\theta} / \delta, & \dot{\theta} \leq \delta .
\end{array} \quad \operatorname{sat}(\dot{\phi})= \begin{cases}1, & \dot{\phi}>\delta \\
\dot{\phi} / \delta, & \dot{\phi} \leq \delta .\end{cases}\right.
$$

The boundary layer of $\omega_{s}$ in this $3 D$ FTSMG is bigger than in the new guidance law proposed.

Proof. Considering Remark 2, when the original commanded accelerations with the saturation function are applied, it is clear that the LOS angular speeds, $\dot{\theta}$ and $\dot{\phi}$ in the pitch and yaw planes are bounded as:

$$
\begin{aligned}
& |\dot{\theta}| \leq \delta \\
& |\dot{\phi}| \leq \delta
\end{aligned}
$$

Since $\omega_{s}=\sqrt{(\dot{\theta} \cos \phi)^{2}+\dot{\phi}^{2}}$, the boundary layer of $\omega_{s}$ in the original 3D FTSMG is obtained as:

$$
\omega_{s}=\sqrt{(\dot{\theta} \cos \phi)^{2}+\dot{\phi}^{2}} \leq \delta \sqrt{(\cos \phi)^{2}+1}
$$

The inequality, $\sqrt{(\cos \phi)^{2}+1} \geq 1$, completes the proof.

If the target acceleration could be precisely measured or estimated, the new proposed 3D FTSMG can be obtained as:

$$
\boldsymbol{a}_{m c}=a_{m \perp} \boldsymbol{e}_{\perp}=\left[-N \dot{r} \omega_{s}+\left(a_{t \perp}+\beta \omega_{s}^{\eta}\right) \operatorname{sat}\left(\omega_{s}\right)\right] \boldsymbol{e}_{\perp} .
$$

For the nonmanoeuvring targets, the commanded acceleration becomes:

$$
\boldsymbol{a}_{m c}=a_{m \perp} \boldsymbol{e}_{\perp}=\left[-N \dot{r} \omega_{s}+\beta \omega_{s}^{\eta} \operatorname{sat}\left(\omega_{s}\right)\right] \boldsymbol{e}_{\perp} .
$$

As shown in Eqn. (50), the commanded acceleration consists of three terms: $-N \dot{r} \omega_{s}$ is the proportional navigation term, $f_{\perp} \operatorname{sat}\left(\omega_{s}\right)$ the variable structure term to cope with the target manoeuvre, and $\beta \omega_{s}^{\eta}$ sat $\left(\omega_{s}\right)$ the finite convergent term. If $\eta=1$, the finite convergent term becomes a contribution to the proportional navigation term. On the other 
TABLE I

INITIAL CONDITIONS OF THE INTERCEPTOR AND TARGET IN CASE 1 AND CASE 2

\begin{tabular}{|c|c|c|}
\hline & Interceptor & Target \\
\hline Velocity $(\mathrm{m} / \mathrm{s})$ & 500 & 1000 \\
\hline Heading angle (deg) & 10 & 225 \\
\hline Flight path angle (deg) & 50 & -45 \\
\hline Relative distance $(\mathrm{km})$ & 10 & \\
\hline Elevation angle (deg) & 45 & \\
\hline Azimuth angle (deg) & 45 & \\
\hline
\end{tabular}

hand, if $\eta=0$, the finite convergent term is transformed into a variable structure term and the proposed 3D FTSMG becomes a first-order 3D sliding mode guidance law. The commanded acceleration for $\eta=0$ is given by:

$$
\boldsymbol{a}_{m c}=a_{m \perp} \boldsymbol{e}_{\perp}=\left[-N \dot{r} \omega_{s}+f_{\perp}^{\prime} \operatorname{sat}\left(\omega_{s}\right)\right] \boldsymbol{e}_{\perp}
$$

where $f_{\perp}^{\prime}=f_{\perp}+\beta$. If the gain of the variable structure term, $f_{\perp}^{\prime}$, is bigger than the maximum target acceleration component, the first-order 3D sliding mode guidance law also guarantees the convergence of the LOS rate in a finite time. In this sliding mode guidance law, the convergence speed is proportional to the gain of the variable structure term.

\section{Numerical Simulations}

This section demonstrates the performance and properties of the proposed approach through numerical simulations. For rigorous verification, the performance of the the proposed 3D FTSMG is compared with that of the 3D FTSMG developed in Ref. [3].

In the engagement scenarios, the east-north-up (ENU) frame is used as the reference frame. The initial states of the interceptor and target are given in Table I. Note that, as shown in Table I, the initial elevation and azimuth angle and their initial rates can be readily calculated: $\theta_{0}=45 \mathrm{deg}, \phi_{0}=45 \mathrm{deg}, \dot{\theta}_{0} \approx 0.4852 \mathrm{deg} / \mathrm{s}$, and $\dot{\phi}_{0} \approx 1.5062 \mathrm{deg} / \mathrm{s}$. Since these values are significant to be ignored, there exist the cross coupling effect between the pitch and yaw loops of the spherical LOS coordinate system.

Both the guidance laws use a saturation function rather than the signum function to avoid the chattering issue. In the saturation function, we set $\delta=0.05 \mathrm{deg} / \mathrm{s}$. As explained, $N$ in the guidance command is the PNG component and the navigation gain is typically set to be between 3 and 5 . In both the original and new guidance laws, $N=3$. A few combination of the other design parameters $\beta$ and $\eta$ are investigated and several cases are considered in the the benchmark scenario.

In all simulation cases, the total acceleration bound of target manoeuvres is set to be equal to $20 \sqrt{2} \mathrm{~m} / \mathrm{s}^{2}$ and it is assumed that this bound can be estimated. Therefore, for the previous FTSMG, both $f_{\theta}, f_{\phi}$, and $f_{\perp}$ are set to be identical to $20 \sqrt{2} \mathrm{~m} / \mathrm{s}^{2}$. 


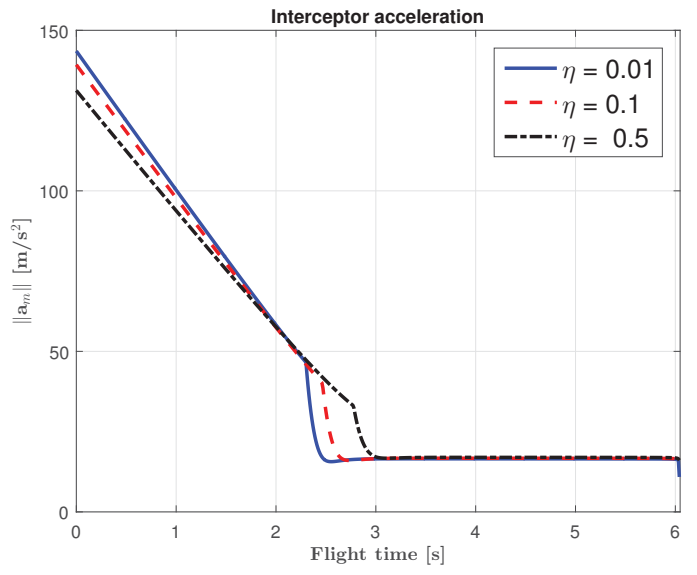

(a) Total commanded acceleration

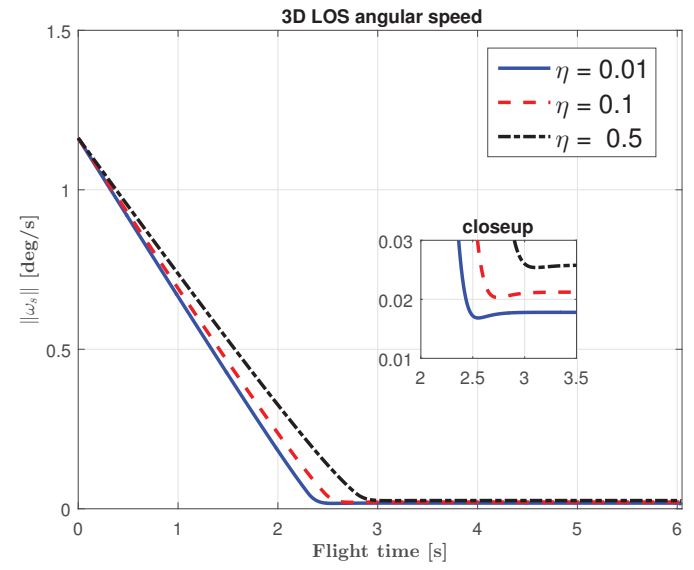

(b) LOS angular speed

Fig. 2. Total commanded acceleration and LOS angular speed in case $1(\beta=15)$

Combinations of the two design parameters $\beta$ and $\eta$ divide the two cases. The target acceleration vector in its body axis under these two cases is given as $\boldsymbol{a}_{t}^{b}=g[0,0.5,1.5]^{T}$ where $g$ denotes the gravitational accleration and the superscript $b$ means that the vector is represented in the body axis frame.

Case 1: $\beta=20$ and $\eta=0.01,0.1,0.5$. The time histories of the total commanded accelerations and LOS angular speeds are illustrated in Fig. 2. For all the given combinations of the two design parameters, the LOS angular speed converges to the prescribed boundary layer, $\omega_{s}<0.03 \mathrm{deg} / \mathrm{s}$, in a finite time, which coincides with Theorem 1 . The initial values of the LOS angular speeds in case 1 is smaller than $e \mathrm{rad} / \mathrm{s}$ as shown in Fig. 2(b). Therefore, from Remark 1, it is clear that the convergence speed of $\omega_{s}$ is most likely to be decreased as the value of $\eta$ decreases. As depicted in Fig. 2(b), this analysis result coincides with the simulation results in all scenarios.

Now, let us compare the performance of the new 3D FTSMG proposed in this paper with that of the FTSMG in [3]. The miss distance and total control energy required for each scenario are represented in Table II. As shown, the miss distances are smaller than $0.00005 m$ under all the combinations, which implies that the two 3D FTSMG laws successfully intercept the manoeuvring target. It is worth noting that the new 3D FTSMG spends less total control energy in all the scenarios, compared with the 3D FTSMG in [3]. For $\beta=20$ and $\eta=0.01$, the profiles of the total acceleration and LOS angular speed are compared in Fig. 3. Note that all the design parameters are set to be identical in the two guidance laws and flight conditions are also same. Under these conditions, Theorem 2 indicates that the total acceleration of the proposed approach will be smaller compared with the original 3D FTSMG at the beginning of the engagement. Fig. 3(a) demonstrates that the total acceleration of the proposed approach is indeed smaller than that of the original FTSMG at the beginning, but becomes bigger than the other as the flight conditions becomes different. As shown Fig. 3(a), there are two jumps in the total acceleration change of the previous 3D FTSMG, whereas one in the proposed FTSMG. This is because the commanded acceleration vector in the previous 3D FTSMG is obtained by decoupling 3D space into two 2D planes, whereas the one proposed in 
TABLE II

COMPARISONS ON THE MisS DISTANCE AND TOTAL CONTROL ENERGY REQUIRED IN CASE 1

\begin{tabular}{cccc}
\hline \hline & & New 3D FTSMG & 3D FTSMG in [3] \\
\hline \hline$\beta=15$ & miss $(m)$ & 0.00002 & 0.00002 \\
\cline { 2 - 4 }$\eta=0.01$ & energy $\left(\mathrm{m}^{2} / \mathrm{s}^{4}\right)$ & 279.09 & 287.56 \\
\hline$\beta=15$ & miss $(\mathrm{m})$ & 0.00004 & 0.00004 \\
\cline { 2 - 4 }$\eta=0.1$ & energy $\left(\mathrm{m}^{2} / \mathrm{s}^{4}\right)$ & 278.68 & 286.43 \\
\hline$\beta=15$ & miss $(\mathrm{m})$ & 0.00005 & 0.00005 \\
\cline { 2 - 4 }$\eta=0.5$ & energy $\left(\mathrm{m}^{2} / \mathrm{s}^{4}\right)$ & 278.33 & 285.11 \\
\hline
\end{tabular}

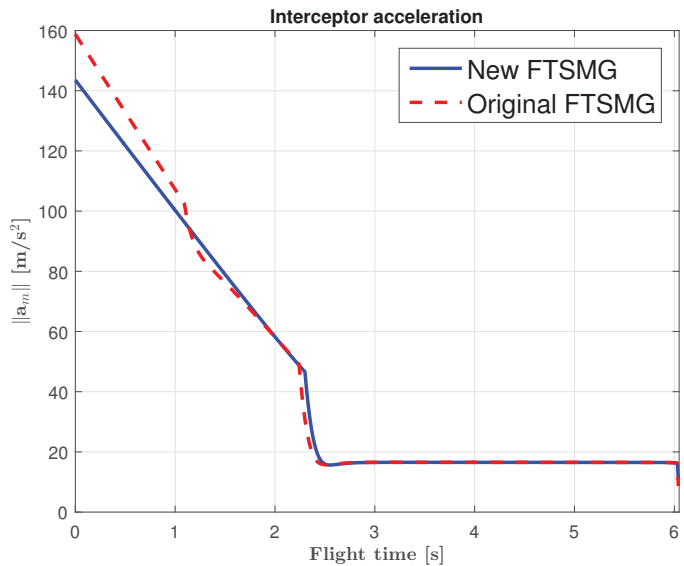

(a) Total commanded acceleration

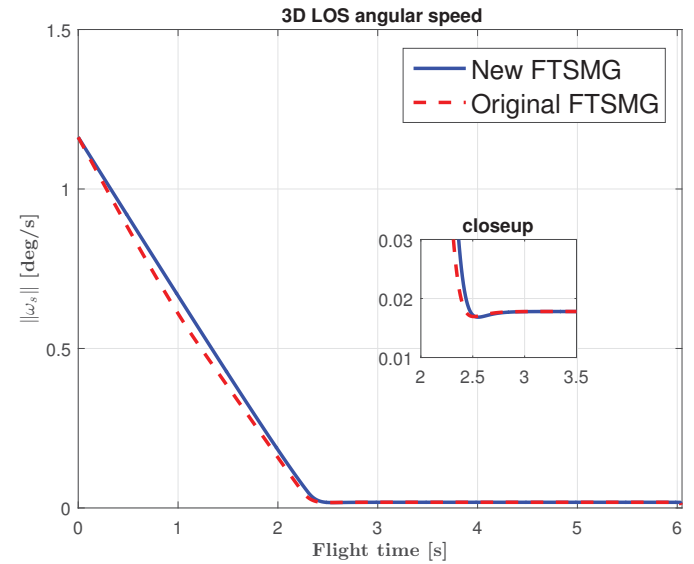

(b) LOS angular speed

Fig. 3. Comparison on the total commanded acceleration and LOS angular speed in case $1(\beta=15$ and $\eta=0.01)$

this paper obtains the acceleration vector directly using the kinematics in 3D space. Fig. 3(b) shows that although the new guidance law spends less energy, the settling time of $\omega_{s}$ is almost identical to that of the previous guidance law. The comparison results confirm that the proposed 3D FTSMG more efficiently nullifies the LOS angular rate, compared with its counterpart 3D FTSMG.

Since the simulation results show identical pattern, illustrations of the comparison results for the other two combinations of $\beta$ and $\eta$ are skipped in this paper.

Case 2: $\beta=5,10,20$ and $\eta=0.02$. For all the combinations of $\beta$ and $\eta$ in case 2, the miss distances in the proposed guidance law are smaller than $0.00004 \mathrm{~m}$. Therefore, the new 3D FTSMG guarantees the interception of the manoeuvring target in a finite time in case 2. The profiles of the total commanded acceleration and 3D LOS rate are depicted in Fig. 4. From the figure, it is clear that $\omega_{s}$ converges a small neighbourhood of zero in a finite time, i.e. Theorem 1 satisfies in case 2. Fig. 4(b) demonstrates that the convergence speed of $\omega_{s}$ increases as $\beta$ increases, which confirms Remark 1. As shown in a closeup in Fig. 4(b), the boundary layer of the LOS angular speeds under all the combinations holds Remark $2, \omega_{s} \leq \delta=0.05 \mathrm{deg} / \mathrm{s}$. 


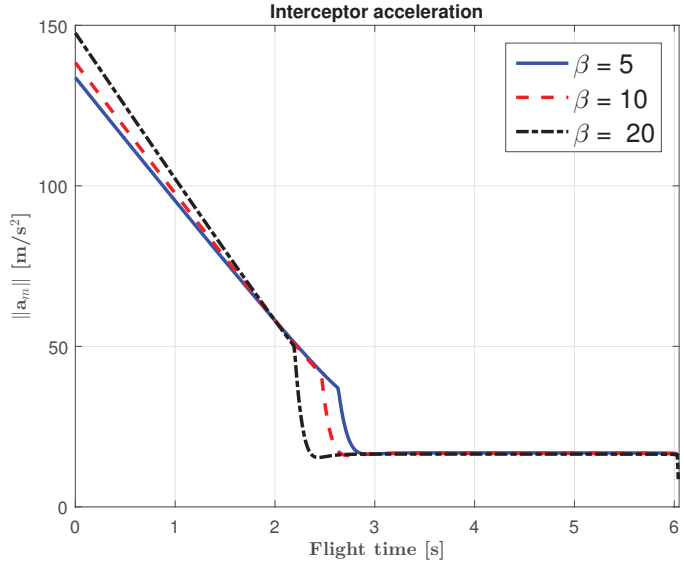

(a) Total commanded acceleration

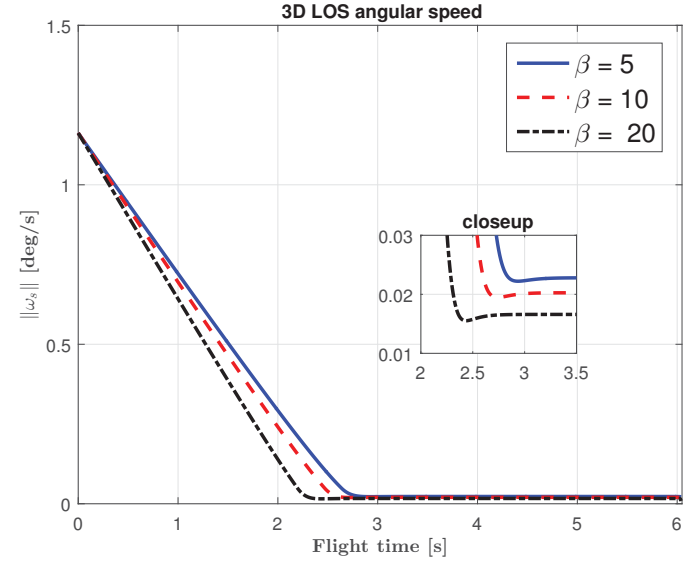

(b) LOS angular speed

Fig. 4. Total commanded acceleration and LOS angular speed in case $2(\eta=0.02)$

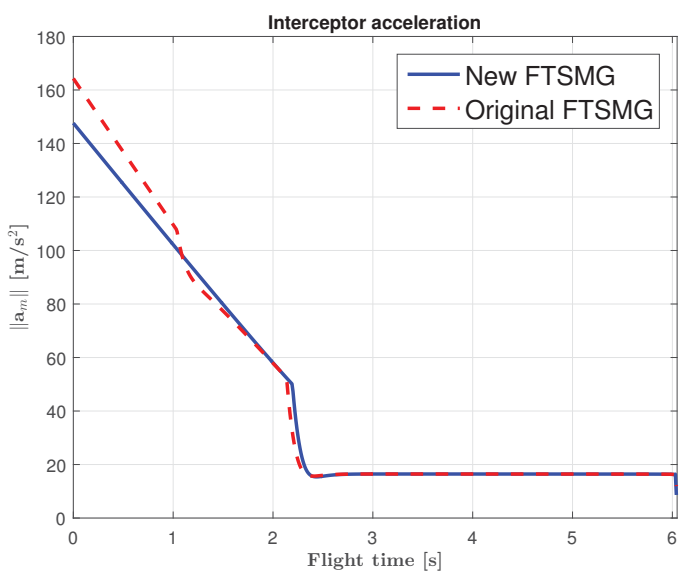

(a) Total commanded acceleration

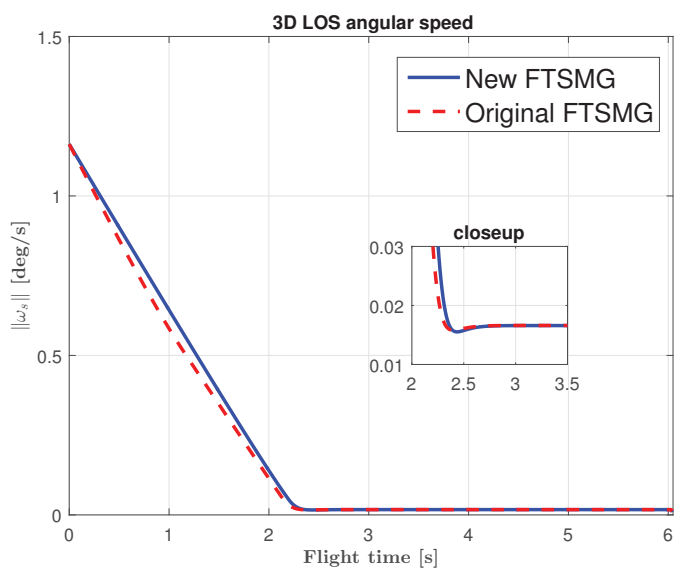

(b) LOS angular speed

Fig. 5. Comparison on the total commanded acceleration and LOS angular speed in Case $2(\beta=20$ and $\eta=0.02)$

Fig. 5 compares the profiles of total energy and LOS angular rate of the two guidance laws for $\beta=20$ and $\eta=0.01$. The comparison results in case 2 are similar to those in case 1 . The miss distance and total control energy consumed in the two guidance laws under case 2 are summarised in Table III.

Like case 1, the comparison results represent that the proposed 3D FTSMG achieves similar miss distances with less total control energy, compared with its counterpart 3D FTSMG. This suggests that the new 3D FTSMG is more efficient in nullifying the LOS angular rate than the previous guidance law.

Case 3 and Case 4. Note that analysis in Section III and Section IV does not consider the maximum acceleration limit. In order to first validate the analysis results through numerical simulations, the maximum acceleration limit was not considered in cases 1 and 2. Then, the numerical simulations with an acceleration limit have been performed 
TABLE III

COMPARISONS ON THE Miss DisTANCE AND TOTAL CONTROL ENERGY REQUiRED IN CASE 2

\begin{tabular}{cccc}
\hline \hline & & New 3D FTSMG & 3D FTSMG in [3] \\
\hline \hline$\beta=5$ & miss $(m)$ & 0.0005 & 0.00004 \\
\cline { 2 - 4 }$\eta=0.02$ & energy $\left(\mathrm{m}^{2} / \mathrm{s}^{4}\right)$ & 278.53 & 285.90 \\
\hline$\beta=10$ & miss $(\mathrm{m})$ & 0.0004 & 0.00003 \\
\cline { 2 - 4 }$\eta=0.02$ & energy $\left(\mathrm{m}^{2} / \mathrm{s}^{4}\right)$ & 278.75 & 286.67 \\
\hline$\beta=20$ & miss $(\mathrm{m})$ & 0.0001 & 0.00002 \\
\cline { 2 - 4 }$\eta=0.02$ & energy $\left(\mathrm{m}^{2} / \mathrm{s}^{4}\right)$ & 279.34 & 288.12 \\
\hline
\end{tabular}

TABLE IV

COMPARISONS ON THE MISS DISTANCE AND TOTAL CONTROL ENERGY REQUIRED IN CASE 3

\begin{tabular}{cccc}
\hline \hline & & New 3D FTSMG & 3D FTSMG in [3] \\
\hline \hline$\beta=15$ & miss $(\mathrm{m})$ & 0.00002 & 0.00002 \\
\cline { 2 - 4 }$\eta=0.01$ & energy $\left(\mathrm{m}^{2} / \mathrm{s}^{4}\right)$ & 289.58 & 301.51 \\
\hline$\beta=15$ & $\operatorname{miss}(\mathrm{m})$ & 0.00001 & 0.00001 \\
\cline { 2 - 4 }$\eta=0.1$ & energy $\left(\mathrm{m}^{2} / \mathrm{s}^{4}\right)$ & 289.20 & 298.95 \\
\hline$\beta=15$ & $\operatorname{miss}(\mathrm{m})$ & 0.00001 & 0.00002 \\
\cline { 2 - 4 }$\eta=0.5$ & energy $\left(\mathrm{m}^{2} / \mathrm{s}^{4}\right)$ & 288.55 & 296.81 \\
\hline
\end{tabular}

for cases 1 and 2. The considered maximum translation accelerations of the interceptor in the elevation and azimuth directions are both equal to the value of $8 g$ where $g \approx 9.8 \mathrm{~m} / \mathrm{s}^{2}$. The corresponding benchmarks to cases 1 and 2 are named as case 3 and case 4. The comparison results are summarised in Tables VI and V. The miss distances are smaller than $0.00021 \mathrm{~m}$ in all the simulations, which confirms that the two guidance laws successfully intercept a manoeuvring target.

Fig. 6 depicts the comparison results between the two guidance laws for $\beta=20$ and $\eta=0.02$. As shown in Fig. 6(a), the total commanded acceleration is limited to the maximum in both guidance laws. Comparing the results represented in Fig. 5, it is evident that their difference in the energy consumptions becomes a bit larger

TABLE V

COMPARISONS ON THE Miss Distance AND total CONTROL ENERGy UNDER CASE 4

\begin{tabular}{cccc}
\hline \hline & & New 3D FTSMG & 3D FTSMG in [3] \\
\hline \hline$\beta=5$ & miss $(m)$ & 0.00001 & 0.00001 \\
\cline { 2 - 4 }$\eta=0.02$ & energy $\left(\mathrm{m}^{2} / \mathrm{s}^{4}\right)$ & 288.82 & 297.96 \\
\hline$\beta=10$ & miss $(\mathrm{m})$ & 0.0001 & 0.00002 \\
\cline { 2 - 4 }$\eta=0.02$ & energy $\left(\mathrm{m}^{2} / \mathrm{s}^{4}\right)$ & 289.19 & 299.18 \\
\hline$\beta=20$ & miss $(\mathrm{m})$ & 0.0021 & 0.00014 \\
\cline { 2 - 4 }$\eta=0.02$ & energy $\left(\mathrm{m}^{2} / \mathrm{s}^{4}\right)$ & 289.83 & 301.33 \\
\hline
\end{tabular}




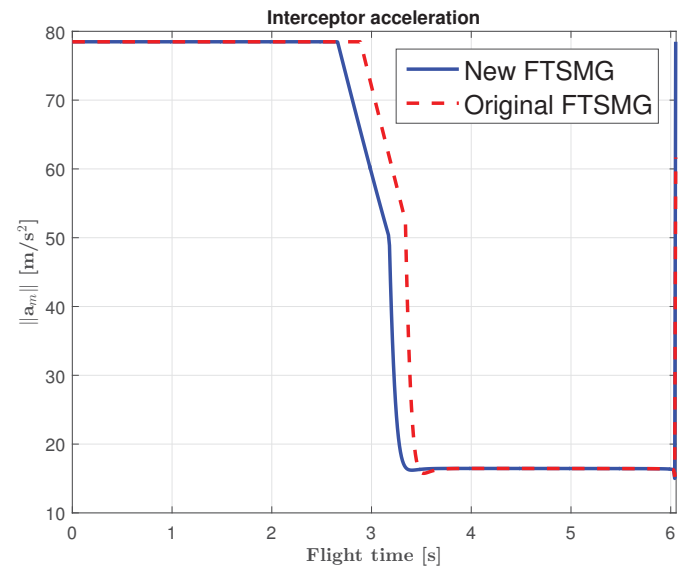

(a) Total commanded acceleration

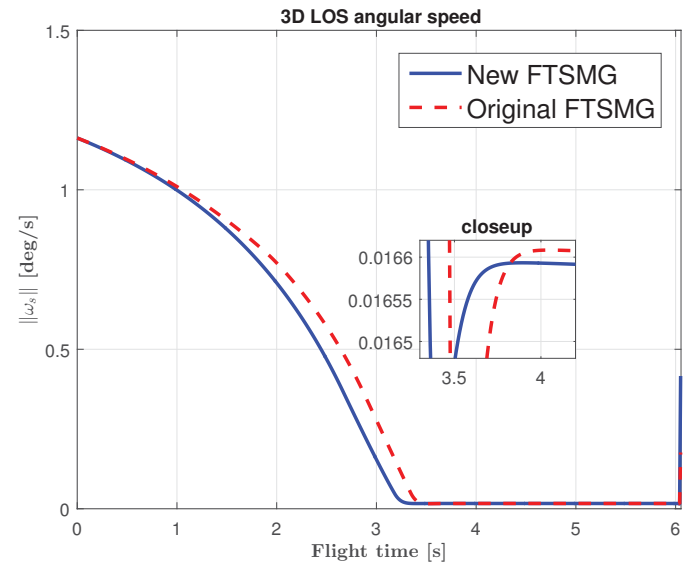

(b) LOS angular speed

Fig. 6. Comparison on the total commanded acceleration and LOS angular speed in case 4 ( $\beta=20$ and $\eta=0.02)$

under the acceleration saturation. It is clearly demonstrated that $\omega_{s}$ in the proposed 3D FTSMG converges faster to a smaller value with less control energy spent, compared with the previous 3D FTSMG. The simulation results confirm that the new 3D FTSMG is nullified more efficiently than in the previous FTSMG under the presence of the acceleration saturation. This implies that the analysis results could be extended to the practical cases where the total acceleration is bounded by its physical limit.

Note that the trend of the simulation results in cases 3 and 4 remain similar in all the scenarios in cases 1 and 2.

Case 5. This section also examines the performance of the guidance laws through an extreme benchmark scenario. The target manoeuvre in case 3 is the identical to that in cases 1 and 2. The initial conditions of the interceptor and target in the second benchmark are also the same as those in cases 1 and 2 except the initial heading angle of the interceptor: it is set to be equal to $-4 d e g$. The initial heading and flight path angles of the interceptor are intentionally chosen far away from the collision course, so that it is expected to require large acceleration commands at the beginning of the engagement. Given the initial geometry conditions, we have: $\theta_{0}=45 \mathrm{deg}, \phi_{0}=45 \mathrm{deg}$, $\dot{\theta}_{0}=1.3898 \mathrm{deg} / \mathrm{s}$, and $\dot{\phi}_{0}=0.6975-\mathrm{deg} / \mathrm{s}$. These values are significant to be ignored and thus the cross coupling effect between the two planes of the spherical LOS coordinate system will become an issue.

The miss distance and total control energy consumed in the two guidance laws are compared in Table VI. Although the original 3D FTSMG spent more total control energy compared with the proposed 3D FTSMG, it cannot intercept the target. On the other hand, the proposed 3D FTSMG successfully intercepts the target.

Fig. 7 compares the total commanded acceleration and LOS angular speed between the two guidance laws. The figure confirms that the 3D FTSMG in [3] cannot successfully nullify the LOS angular rate, even if it applies more control energy than the new 3D FTSMG. As illustrated, the total acceleration of the previous 3D FTSMG is saturated until the end of the engagement. This implies that since the coupling effect is not considered in the 
TABLE VI

COMPARISONS ON THE MisS DisTANCE AND TOTAL CONTROL ENERGY REQUiRED IN CASE 5

\begin{tabular}{cccc}
\hline \hline & & New 3D FTSMG & 3D FTSMG in [3] \\
\hline \hline$\beta=20$ & miss $(m)$ & 0.00005 & 198.493 \\
\cline { 2 - 4 }$\eta=0.02$ & energy $\left(\mathrm{m}^{2} / \mathrm{s}^{4}\right)$ & 455.08 & 485.01 \\
\hline
\end{tabular}

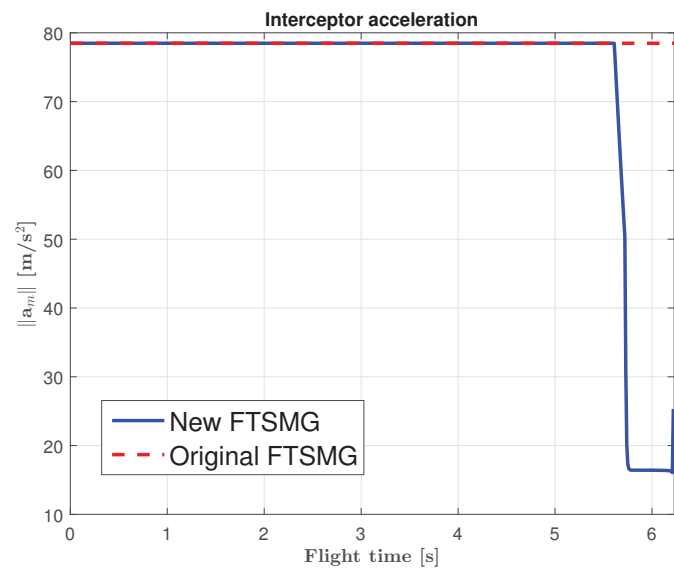

(a) Total commanded acceleration

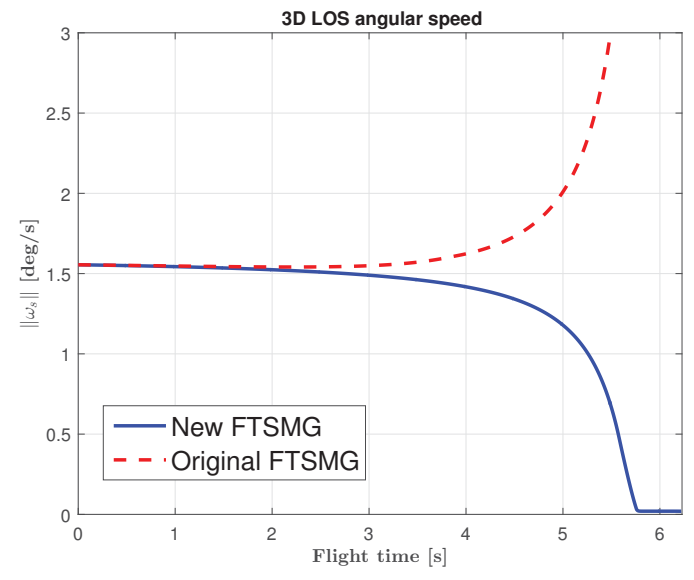

(b) LOS angular speed

Fig. 7. Comparison on the total commanded acceleration and LOS angular speed in Case $5(\beta=20$ and $\eta=0.02)$

guidance design phase, the original 3D FTSMG cannot effectively drive the LOS angular rate to zero or its small neighbourhood in some extreme engagement condition. The comparison results verifies that the energy portion not contributing to the reduction of the LOS angular rate in the proposed FTSMG spend is less than in the 3D FTSMG compared. This also validates the theoretical analysis results.

\section{CONCLUSIONS}

In this paper, a new 3D FTSMG law is proposed. This new guidance law guarantees finite time convergence of the 3D LOS angular rate for the interception of the target in a finite time. The guidance law accepts the concept where nullifying the LOS rate guarantees the interception of the target and its derivation is based on FTSMG presented in Ref. [3]. Since the two guidance loops, elevation and azimuth loops, were separately designed, the cross coupling effect between the two loops were ignored in the previous 3D FTSMG in [3]. If this cross coupling effect is significant, the guidance law might generate accelerations unnecessary in reduction of the LOS rate, i.e. waste energy. In order to alleviate the issue with the cross coupling effect, this paper first establishes the 3D relative kinematic equation set in a rotating LOS coordinate system and then develops a FTSMG using this set. Finite time convergence is theoretical analysed and its characteristics such as settling time are also theoretically compared to those in the original 3D FTSMG. It is proven that, under the same flight conditions with same design parameters, the new 3D guidance law spends less energy than the original 3D FTSMG. In the theoretical analysis, the boundary 
layer of the 3D LOS speed is also proven to be smaller than that in the original guidance law. The performance and properties of the new 3D FTSMG are demonstrated through numerical simulations and it is shown that the simulations results correspond to the theoretical analysis results.

\section{REFERENCES}

[1] Y. B. Shtessel, I. A. Shkolnikov, and A. Levant, "Smooth second-order sliding modes: Missile guidance application," Automatica, vol. 43, no. 8, pp. 1470-1476, 2007.

[2] _ - "Guidance and control of missile interceptor using second-order sliding modes," Aerospace and Electronic Systems, IEEE Transactions on, vol. 45, no. 1, pp. 110-124, 2009.

[3] D. Zhou, S. Sun, and K. L. Teo, "Guidance laws with finite time convergence," Journal of guidance, control, and dynamics, vol. 32, no. 6, pp. 1838-1846, 2009.

[4] D. Zhou, C. Mu, and W. Xu, "Adaptive sliding-mode guidance of a homing missile," Journal of Guidance, Control, and Dynamics, vol. 22, no. 4, pp. 589-594, 1999.

[5] S. Sun, D. Zhou, and W.-T. Hou, "A guidance law with finite time convergence accounting for autopilot lag," Aerospace Science and Technology, vol. 25, no. 1, pp. 132-137, 2013.

[6] S. R. Kumar, S. Rao, and D. Ghose, "Nonsingular terminal sliding mode guidance with impact angle constraints," Journal of Guidance, Control, and Dynamics, pp. 1-17, 2014.

[7] X. Wang and J. Wang, "Partial integrated missile guidance and control with finite time convergence," Journal of Guidance, Control, and Dynamics, vol. 36, no. 5, pp. 1399-1409, 2013.

[8] _ - "Partial integrated guidance and control for missiles with three-dimensional impact angle constraints," Journal of Guidance, Control, and Dynamics, vol. 37, no. 2, pp. 644-657, 2014.

[9] C.-D. Yang and C.-C. Yang, "Analytical solution of three-dimensional realistic true proportional navigation," Journal of guidance, control, and dynamics, vol. 19, no. 3, pp. 569-577, 1996.

[10] — " "Analytical solution of generalized three-dimensional proportional navigation," Journal of guidance, control, and dynamics, vol. 19, no. 3, pp. 721-724, 1996.

[11] — , "Analytical solution of 3d true proportional navigation," Aerospace and Electronic Systems, IEEE Transactions on, vol. 32, no. 4, pp. 1509-1522, 1996.

[12] N. A. Shneydor, Missile guidance and pursuit: kinematics, dynamics and control. Elsevier, 1998.

[13] C. Li, W. Jing, H. Wang, and Z. Qi, "Gain-varying guidance algorithm using differential geometric guidance command," Aerospace and Electronic Systems, IEEE Transactions on, vol. 46, no. 2, pp. 725-736, 2010.

[14] K. Li, L. Chen, and X. Bai, "Differential geometric modeling of guidance problem for interceptors," Science China Technological Sciences, vol. 54, no. 9, pp. 2283-2295, 2011.

[15] K. Li, L. Chen, and G. Tang, "Improved differential geometric guidance commands for endoatmospheric interception of high-speed targets," Science China Technological Sciences, vol. 56, no. 2, pp. 518-528, 2013.

[16] F. Tyan, "Unified approach to missile guidance laws: A 3d extension," Aerospace and Electronic Systems, IEEE Transactions on, vol. 41, no. 4, pp. 1178-1199, 2005.

[17] — - "Capture region of a gipn guidance law for missile and target with bounded maneuverability," Aerospace and Electronic Systems, IEEE Transactions on, vol. 47, no. 1, pp. 201-213, 2011.

[18] K. Li, T. Zhang, and L. Chen, "Ideal proportional navigation for exoatmospheric interception," Chinese Journal of Aeronautics, vol. 26, no. 4, pp. 976-985, 2013. 
2017-03-30

\title{
A new three-dimensional sliding mode guidance law variation with finite time convergence
}

\author{
Shin, Hyo-Sang
}

Institute of Electrical and Electronics Engineers

Hyo-Sang Shin, Ke-Bo Li and Antonios Tsourdos. A new three-dimensional sliding mode guidance law variation with finite time convergence. Available online 30 March 2017

http://dx.doi.org/10.1109/TAES.2017.2689938

Downloaded from Cranfield Library Services E-Repository 